Military Technical College Kobry El-Kobbah, Cairo, Egypt

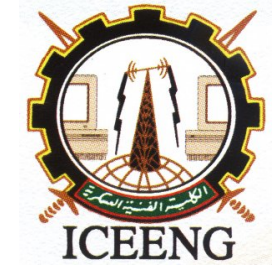

\author{
$7^{\text {th }}$ International Conference \\ on Electrical Engineering \\ ICEENG 2010
}

\title{
Stability Improvement of Distribution Networks using Inverter-based Distributed Generators at different penetration levels
}

\author{
By \\ M. H. Solim, M. I. Marei \\ M. M. Mansour, senior \\ member, IEEE
A. M. A. Mahmoud, member, IEEE

\section{$\underline{\text { Abstract: }}$}

Distributed Generators are rapidly penetrating the traditional Power Distribution Networks, offering a number of technical, environmental and economic benefits. Meanwhile, they have some negative impacts on network stability as they noticeably reduce the overall rotational inertia and then the utility will no longer be an Infinite Bus having constant voltage and frequency. Therefore DG's have to improve system stability in order to have an efficient and, in the same time, a robust network. In order to optimally utilize DG's, they need to be dispatchable, which is not always an available facility. This paper utilizes a multi-function nonlinear inverter-based link for DG systems, Flexible Distribution Generation, to implement several ancillary services and improve system performance depending on their penetration level in the network. The performance of the proposed system is simulated using PSCAD/EMTDC. The results are discussed to demonstrate the potential of the proposed scheme.

\section{Keywords:}

Distributed Generation, Frequency control/stability, Micro-grids, Power oscillations, Power system dynamics, PSCAD/EMTDC, Voltage regulation/stability.

\section{Nomenclature}

DG Distributed Generation

FDG Flexible Distributed Generation

PCC Point of Common Coupling

VSI Voltage Source Inverter

QCC Quality Control Center

HCC Hysteresis Current Control 
PLL Phase Locked Loop

THD Total Harmonic Distortion

$\begin{aligned} * & \text { Egyptian Armed Forces } \\ * * & \text { Benha High Technology Institute, Benha, Egypt } \\ * * * & \text { College of Engineering, Cairo University, Cairo, Egypt }\end{aligned}$

\section{Introduction:}

Distributed Generation (DG) system is defined as an electric power source connected directly to the distribution network. This source can be a Co-generation system with micro turbine, Fuel cell generation system or any kind of renewable energy sources. Many studies indicate that DG will play a significant role in the future power system structure [1]. The integration of DG with the utility distribution network offers a number of technical, environmental and economic benefits. Installation of DG's comes in accordance with the trend of liberalization of energy markets aiming at decreasing the cost associated with electrical power transmission and distribution. Supplying loads partially from relatively near sources leads to noticeable reduction of losses and improvement of efficiency, which gives a great opportunity for distribution utilities to improve the performance of networks. Many of DG new technologies do not use a conventional grid coupled synchronous generator to convert primary energy into electricity. Instead, they use a squirrel cage or doubly fed induction generator (some wind turbine concepts) or a synchronous or squirrel cage induction generator that is grid connected through a power electronic converter (other wind turbine concepts, small scale high speed gas turbines). In the case of solar panels and fuel cells, it is not even mechanical power that is converted into electricity. A further difference between these new technologies and conventional means of electrical power generation is that many of them are of considerably smaller size than conventional thermal, nuclear and hydro units that up to this moment deliver the majority of the electrical power consumed worldwide. Therefore, they are often connected to low and medium voltage grids and not to the high voltage transmission grid, resulting in longer electrical distances [2]

When many small non-synchronous generation units replace a significant part of the synchronous power generation capacity, the total rotational inertia of the synchronous generators is decreased significantly. On the other hand, DG's normally have relatively small inertia constant, as they Often utilize non-rotating power sources. As more and more DG's are connected to the system, the reserve capacity of centralized utility 
generation will be reduced for economical consideration. This leads simply to significant reduction of the overall inertia of the network and hence reducing its robustness. As a consequence, the variation in the rotational speed of the synchronous generators due to changes in their net load will become much higher than at present. This causes large frequency variations that can end up in an unstable and insecure grid. This is why future DG systems have to take a dominant part in maintaining stability of the network [3], [4]. The impact and control scope of ancillary services provided by DG on the power system can be local or global (system-wide), depending on their penetration level in the network. Local ancillary services include voltage regulation, reactive power supply and losses reduction, provided and controlled locally where needed. Global (system-wide) ancillary services include frequency control and active power reserves (spinning or standby); which have an impact on the whole system and can be provided anywhere in the power system [5]. As long as the penetration level of these new technologies in power systems is still low, they only cover a minor fraction of the system load. Therefore, in power system dynamics and transient stability studies, they are normally considered as just negative loads [2]. However, Increasing levels of DG are continually replacing large Synchronous Generators (SG) as the dominating generation technology. On one hand, stiff inverter-based generation technologies seem to improve system stability. On the other hand, the increasing electrical distances between synchronous generators in operation and the reduced system inertia will impede the system stability [6]. In order to optimally utilize DGs, they need to be dispatchable; otherwise load switching and frequency drops can not be compensated and losses will not be minimized in the network. Technologies like wind and solar energy which have non-dispatchable characteristics may cause instability and degrade efficiency of future energy systems unless these technologies are equipped with some advanced mechanical and power electronic controllers [7]. This paper proposes an extension to the functionalities of the Flexible Distributed Generation (FDG), mentioned in [1], to damp frequency oscillations and stabilize the distribution network. Current controlled Voltage Source Inverter (VSI) is used for interfacing DG and distribution networks. The command signal for the VSI, which is current signal in nature, will include the information of active power supplied from DG system to compensate the frequency (in case of high penetration level), or simply to minimize losses (in case of low penetration level), and also the reactive power required to compensate the voltage fluctuation at load-side or total reactive power compensation to allow unity power factor at load-side. Current controlled VSI was selected for this function for its fast dynamic response, accurate performance, ease of implementation and its inherent closed loop control for current to guarantee working at the required operating point. 


\section{Frequency and Voltage Stability:}

Due to economic considerations, the electrical power networks are commonly operating near the stability limits. Therefore, oscillatory stability is attracting more interest and its analysis is essential for power system security. Frequency stability refers to the ability of electrical power systems to maintain fixed frequency after being subjected to a severe disturbance. Frequency will not cause a stability problem if the equilibrium between generation and load is restored. This requires sufficient generation reserve and adequate response from the control and protection devices [4]. Employing DG leads to a lower degree of dependence on central generation (Utility); leading to less reserve required. Despite achieving the advantage of noticeable cost reduction, this results in reduction of stability. Reactive power compensation is also an important issue in the control of distribution systems. Reactive current increases the distribution system losses, reduces the system power factor, shrink the active power capability and can cause large-amplitude variations in the load-side voltage. Moreover, rapid changes in the reactive power consumption of large loads can cause voltage amplitude oscillations (e.g., flicker in the case of arc furnaces). This might lead to a change in the electric system real power demand resulting in power oscillations [1]. The introduction of DG to distribution systems can significantly impact the flow of power and voltage conditions at customers and utility equipment.

\section{System Under Study:}

As stated earlier, DG penetration levels are expected to grow fast in the next decades. Talking about expected penetration levels of (20-30\%), or even higher [3], the utility grid can no longer be considered as an infinite bus. This applies also in case of microgrid that is highly penetrated with DG's, where a part of the network is islanded and its loads are supplied only from the local sources (SG or DG).In that case the utility will be simulated as a large Synchronous Generator supplying the large part of the load, but represented by its swing equation(1) rather than the common Infinite Bus producing constant voltage and frequency regardless of the load.

$$
P_{m}-P_{e}=\frac{H}{\pi f_{o}} \frac{\partial^{2} \delta}{\partial t^{2}}+D \frac{\partial \delta}{\partial t}
$$


$P_{m}$ Input mechanical power p.u.

$P_{f}$ Output electrical power p.u.

$\mathrm{H}$ Inertia constant (sec)

fo Nominal frequency $(\mathrm{Hz})$

$\delta \quad$ Power angle (rad)

\section{Damping coefficient}

DG sources have then to work together using appropriate control systems and efficient connections through the internet in a way that enables them to compensate power oscillations in the system forming what we can call a (Virtual Utility) or (Micro Grid). Such coordination can be accomplished through several systems. Among them, for example is FRIENDS (Flexible, Reliable and Intelligent Electrical Energy Delivery System) utilizing its key component, Quality Control Center (QCC). QCC is responsible for coordinating different kinds of energy sources to satisfy consumer needs. Among these sources DG's are certainly introduced [9]. FDG is then proposed as a good link.

In FDG, Distributed Generators are connected to the network through a DC link with a control system that makes the interfacing between the grid and DG flexible. The main function of that link is to regulate the active power supplied from DG to the utility. Besides, the link can also supply reactive power to the utility to enable unity power factor operation or to regulate the voltage at the point of common coupling (PCC) [8].The control system proposed to FDG is shown in Fig (1), connected to a typical power system. The converter manages the amount of current Ic injected to the utility. Utilizing Inverse Park's Transformation, the load current IL is converted from the three phase coordinates to a synchronously rotating frame, where $\Theta$ is the instantaneous angle of the PCC voltage vector obtained from Phasor Locked Loop (PLL) circuit. The quadrature component of the reference current (Iq *) of VSI is set by the voltage control loop through a PI controller so as to keep the voltage of PCC at constant level [8], while the direct component of the reference current (Id *) of VSI can be set so as to deliver the maximum power that DG can afford in order to minimize losses in the network or simply to keep it at any desired value. This is possible at any penetration level, under constraint of DG capacity. At high penetration levels, it can be set to regulate frequency at constant nominal value $(50 \mathrm{~Hz})$. Hysteresis Current Control (HCC) switching technique is utilized for current control of the Voltage Source Inverter (VSI). It very well suits the given application, as there is a lot of current dynamics through the distribution systems, in addition to its insensitivity to distribution network 
parameters variations.

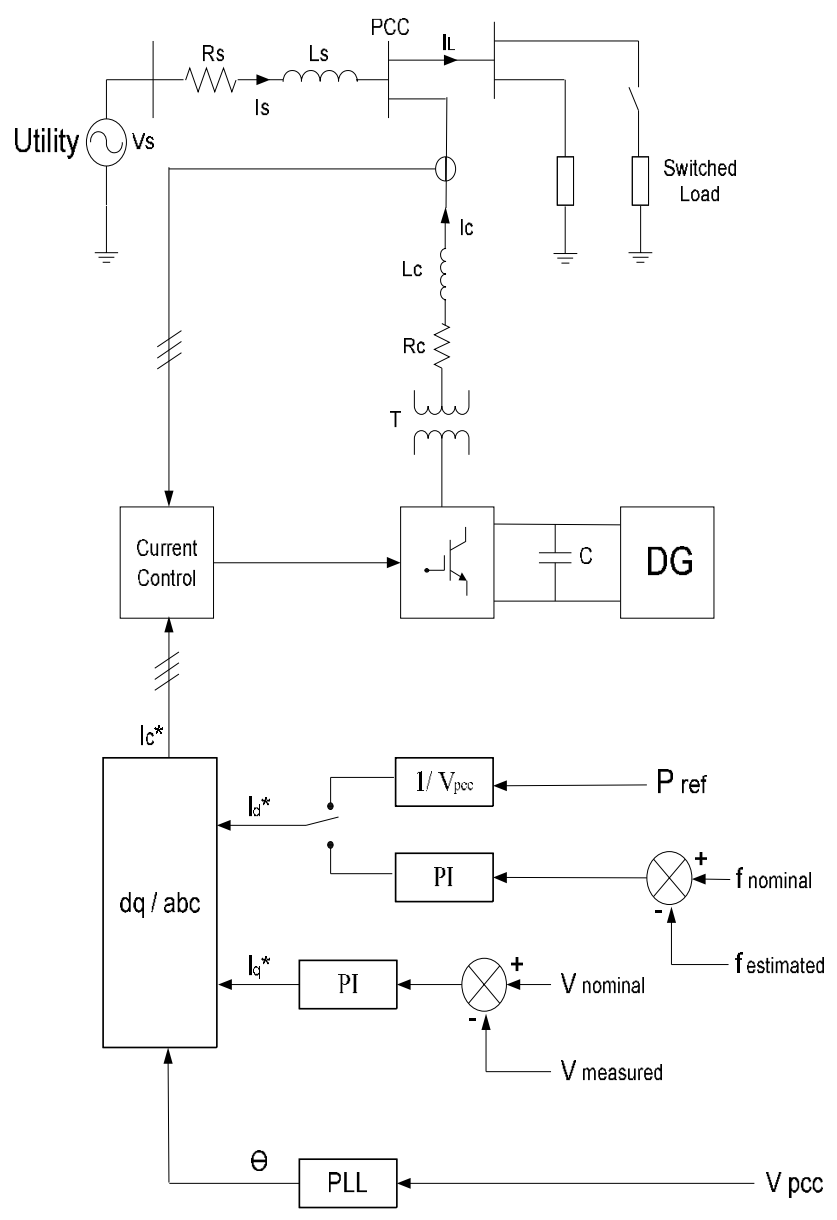

Figure (1): Block diagram of the proposed control system

\section{Power System Frequency Estimation:}

Estimation of power system frequency is an important task since the frequency deviation value is a good indicator of the system abnormal operating conditions. Figure (2) shows the algorithm used for power system frequency estimation, applying orthogonal signal components, (vs and vc), obtained with use of two orthogonal Finite Impulse Response (FIR) filters [10]. The algorithm applied is immune to both signal orthogonal component magnitudes and FIR filter gains variations (equation 2). Several tests to this algorithm have been carried out to verify its capability to track the system frequency with acceptable accuracy, even in transient intervals. 


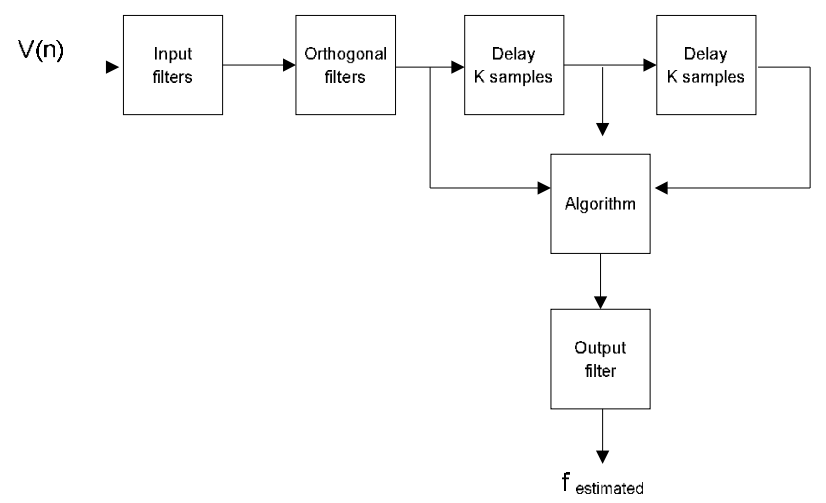

Figure (2): Block diagram of the proposed frequency estimation algorithm

$f=f_{0}\left(1-\frac{1}{\pi} \frac{v_{s}(n) v_{c}(n-2 k)-v_{S}(n) v_{s}(n-2 k)}{v_{g}(n) v_{c}(n-k)-v i(n) v_{s}(n-k)}\right)$

$f$ estimated frequency

$f_{o}$ nominal frequency

\section{Simulated Results:}

The performance of FDG is evaluated by computer simulation using PSCAD/EMTDC. Parameters of the system under study are given in the appendix. The hysteresis band is roughly set to $4 \%$ from the rated value of the VSI current. The following simulation results illustrate the operation of FDG in two selected levels of penetration.

\section{Case (1): Low Penetration level}

In this case the utility is considered an Infinite Bus having constant voltage and frequency. The main task of DG is to keep the voltage constant at load terminals by compensation of reactive power. Figure (3) shows how DG regulates the voltage achieving voltage stability. Voltage profile is flat with error of less than $0.3 \%$. Variations of reactive power demand are almost totally compensated by DG. The command of active power was set at the maximum value DG can supply which reduces losses as much as possible. Changes in active power demand are therefore supplied by the Utility. In that sense DG has no contribution to frequency stability and frequency is kept constant as the Utility is considered an infinite bus. Clearly, control loops of active and reactive power are mutually independent. 

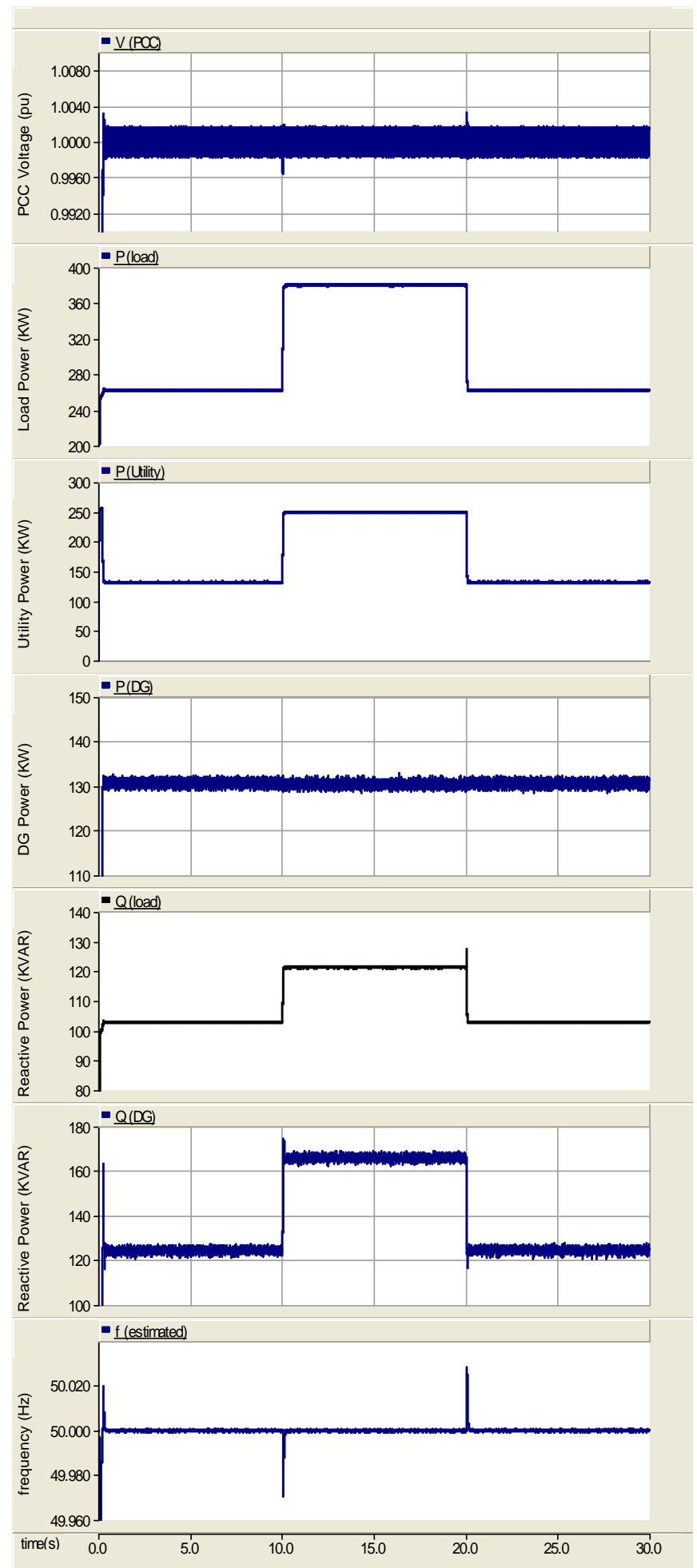

Figure (3): Case (1) Active Power control mode.

(a) Voltage at PCC, (b) Load Power,

(c) Utility Power, (d) DG Power, (e) Load Reactive power,

(f) DG Reactive power, ( $g$ ) Estimated Frequency. 
Case (2): High Penetration level

Now the utility is no longer an infinite bus. It responds to load changes by supplying an amount of its stored kinetic energy leading to frequency variations according to the swing equation. If no appropriate frequency control is involved, it will drop significantly in case of load variations (Fig 4), where a frequency drop of about $1.5 \mathrm{~Hz}$, a totally unaccepted deviation, is produced by a sudden switched load. Therefore, DG has to contribute to frequency regulation as well as keeping the voltage at load terminals.

Figure (5) shows how FDG compensates the load active power variation (about $30 \%$ additional, sudden switched, load ) to keep the frequency at its nominal value, hence achieving frequency stability. It took about 4 seconds to restore frequency at nominal value, without overloading the utility. Meanwhile, voltage is still at flat profile and hence achieving voltage stability and the reactive variation is compensated by FDG.

Figure (6) shows comparison of actual and estimated frequency. In steady state, both are exactly the same. In transient period, they are in phase with a slight difference in magnitude (less than $0.3 \mathrm{~Hz}$ ). Figure (7) shows the load current, DG current, Utility current and phase voltage at PCC at peak load period. It is clear that the Supply voltage and current are almost in phase; reactive power is mainly supplied by FDG. Figure (8) shows the THD of Voltage at PCC, which is below $1 \%$ at all intervals.

The command of active power was set so as to eliminate frequency deviations. This implies the necessity of installing DG's with sufficient reserve capacity on the network, Instead of depending on the reserve capacity of the utility. Tripping delay of DG protection should be somehow longer to allow FDG to compensate power oscillations. If such high DG penetration levels could achieve network stability according to the shown scenario, we would obtain the so called "Virtual Utility". Control loops of active and reactive power are still mutually independent. 


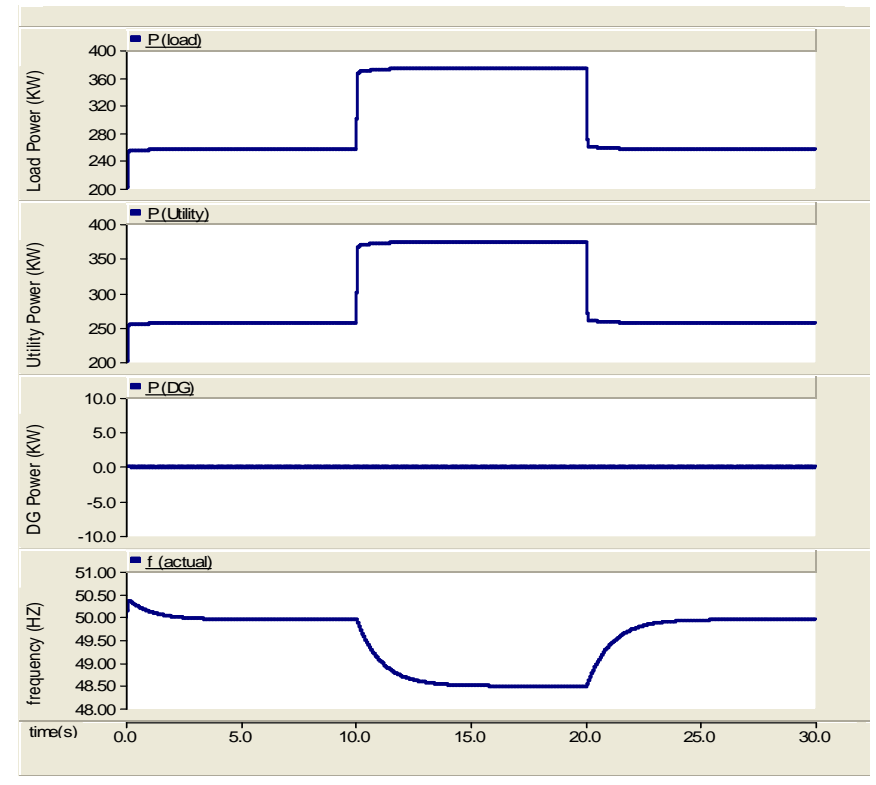

Figure (4): Case (2) System without Frequency Control contribution of FDG (a) Load Power, (b) Utility Power, (c) DG Power, (d) Actual Frequency. 


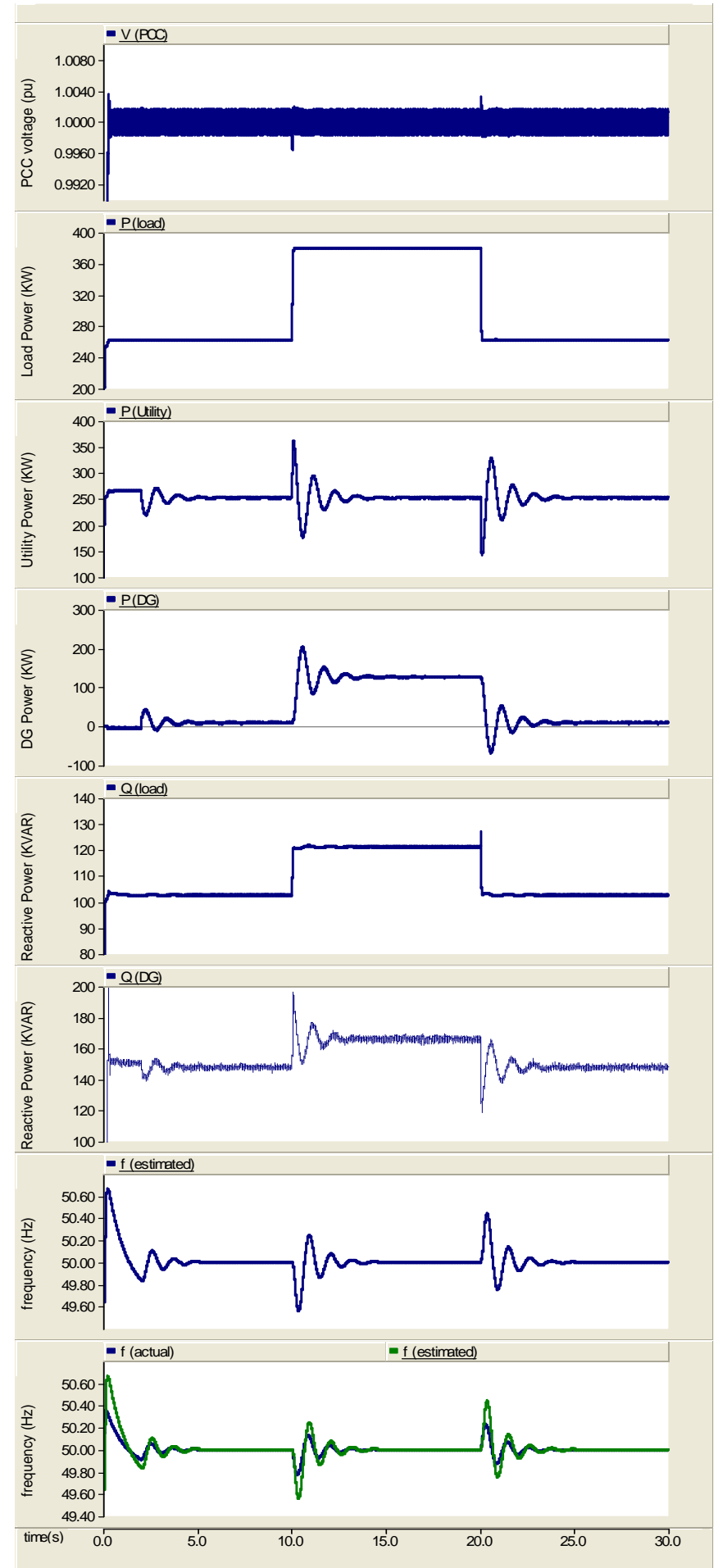

Figure (5): Case (2) Frequency Control mode.

(a) Voltage at PCC, (b) Load Power (\%),

(c) Utility Power (\%), (d) DG Power (\%), (e) Load Reactive power,

(f) DG Reactive power, $(g)$ Estimated Frequency,

(h) Estimated Frequency compared to actual Frequency. 


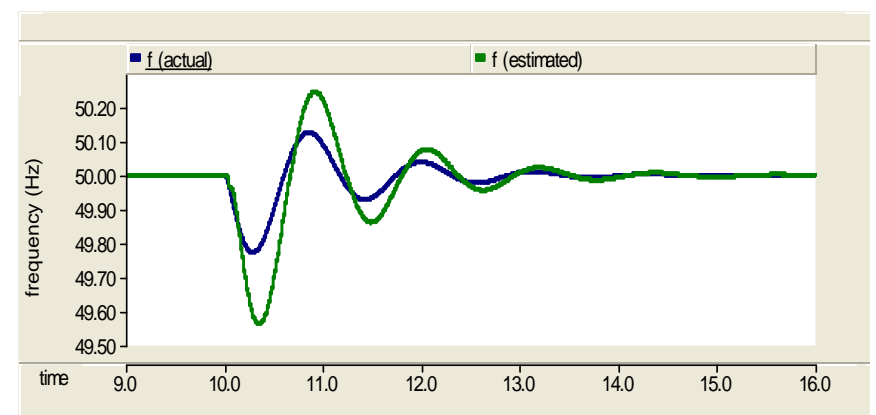

Figure (6): Estimated Frequency compared to actual Frequency

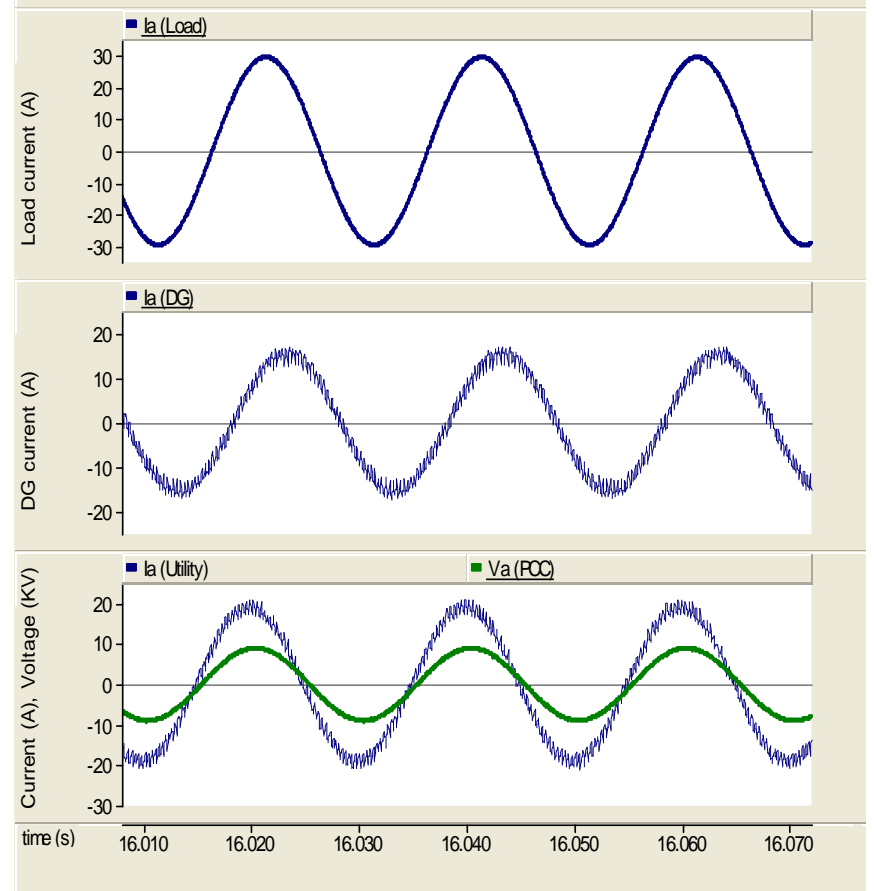

Figure (7): Currents during peak Load.

(a) Load Current (A), (b) DG Current (A),

(c) Voltage at PCC $(K V)$ with Utility Current $(A)$. 


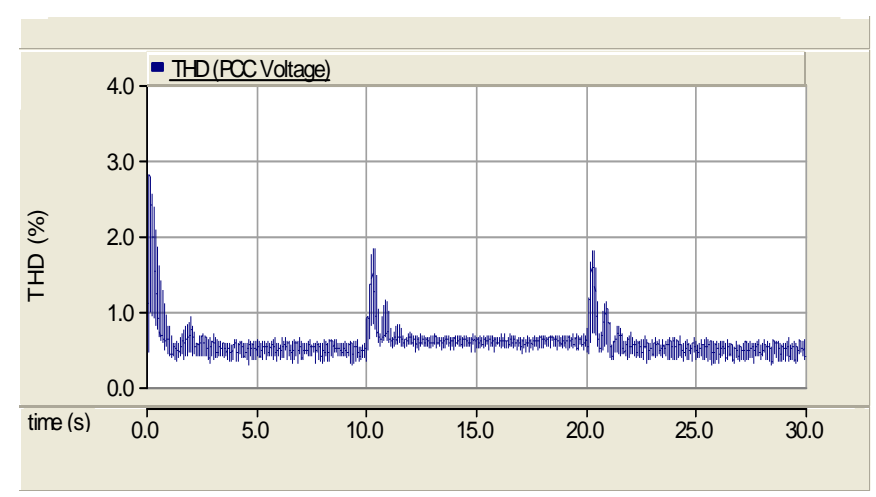

Figure (8): Total Harmonic Distortion of Voltage at PCC.

\section{Conclusions:}

This paper discussed a proposed contribution of DG to stability improvement of Power systems in the near future. As DG improves efficiency, it reduces the overall rotational inertia power reserve. Therefore it has to contribute to both Frequency and Voltage stability. Flexible Distributed Generation FDG sounds to be a good solution for the utility to mitigate several stability problems. The Utility was considered as an Infinite Bus once, and again as a large Synchronous Generator modeled by its Swing Equation. Simulation results show that FDG can improve network performance through different ancillary services; Voltage regulation, Losses reduction and frequency stability. FDG could switch flexibly between these several regulating functions, according to the penetration level of DG in the Network. The fast dynamic response of the proposed system in compensating both active and reactive power at the PCC, achieving full regulation of Voltage and Frequency, presents it as a promising part of future power grids.

\section{Appendix:}

Vs $(\mathrm{rms} /$ Line $)=11 \mathrm{KV}, \mathrm{fo}=50 \mathrm{~Hz}, \mathrm{Ls}=31 \mathrm{mH}, \mathrm{Rs}=1.74 \mathrm{ohm}$.

\section{References:}

[1] M. I. Marei, E. F. El-Saadany and M. M. A. Salama, "Flexible Distributed Generation: (FDG)", Power Engineering Society Summer Meeting, 2002 IEEE, Volume 1, 25-25 July 2002 Page(s):49 - 53 vol.1. 
[2] Slootweg, J.G.; Kling, W.L.; "Impacts of distributed generation on power system transient stability", Power Engineering Society Summer Meeting, 2002 IEEE, Volume 2, 25-25 July 2002 Page(s):862 - 867 vol.2.

[3] Driesen, J.; Visscher, K.; "Virtual synchronous generators", Power, 20-24 July 2008 Page(s): 1 - 3.

[4] Azmy, A.M.; Erlich, "I.;Impact of distributed generation on the stability of electrical power system", Power Engineering Society General Meeting, 2005. IEEE , 12-16 June 2005 Page(s):1056 - 1063 Vol. 2.

[5] Vu Van Thong, Driesen, J. and Belmans, R.; "Benefits and Impact of Using Small Generators for Network Support", Power Engineering Society General Meeting, 2007. IEEE 24-28 June 2007 Page(s): 1 - 7.

[6] Boemer, J.C.; Gibescu, M.; Kling, W.L.; "Dynamic models for transient stability analysis of transmission and distribution systems with distributed generation: An overview" PowerTech, 2009 IEEE Bucharest June 28 2009-July 22009 Page(s): 1 - 8.

[7] Nazari, M.H.; Ilic, M.; "Technical challenges in modernizing distribution electric power systems with large number of distributed generators", PowerTech, 2009 IEEE Bucharest, June 28 2009-July 22009 Page(s): 1 - 8.

[8] Marei, M.I.; El-Saadany, E.F.; Salama, M.M.A.; "A novel control algorithm for the DG interface to mitigate power quality problems", IEEE Transactions on Power Delivery, Volume 19, Issue 3, July 2004 Page(s):1384 - 1392.

[9] Nara, K., "Enabler of introducing distributed generators and its effect to power distribution systems"; Transmission and Distribution Conference and Exhibition 2002: Asia Pacific. IEEE/PES, Volume 1, 6-10 Oct. 2002 Page(s):584 - 589 vol.1.

[10] Szafran, J.; Rebizant, W.; "Power system frequency estimation", Generation, Transmission and Distribution, IEE ProceedingsVolume 145, Issue 5, Sept. 1998 Page(s):578 - 582.

[11] Schauder, C.D.; Gyugyi, L.; Lund, M.R.; Hamai, D.M.; Rietman, T.R.; Torgerson, D.R.; Edris, A.; "Operation of the unified power flow controller (UPFC) under practical constraints", IEEE Transactions on Power Delivery, Volume 13, Issue 2, April 1998 Page(s):630 - 639. 